\title{
Zusammenfassung der ERC-Leitlinien zur Kinderreanimation 2021
}

In den am 24.03.2021 vom ERC veröffentlichten neuen Leitlinien zum Paediatric Life Support [1] hat sich gegenüber der Vorgängerversion von 2015 nur wenig geändert. Dies liegt u.a. auch daran, dass die Leitlinienautoren die seit Jahren vertrauten und trainierten Handlungsabläufe nicht ohne guten Grund verändern wollten.

\section{Was ist neu?}

Das PLS-Leitlinienkapitel ist deutlich umfangreicher, auch weil - in Anlehnung an die Systematik des EPALS-Kurses - zunächst die Beurteilung und Behandlung des kritisch kranken oder verletzten Kindes besprochen wird. Hier wird die Bedeutung der ABCDE-Systematik bekräftigt und das bewährte Pädiatrische Beurteilungsdreieck (Paediatric Assessment Triangle, PAT) als Hilfe zur Erstbeurteilung neu eingeführt. Manche mögen die Angabe von altersentsprechenden Normal- und Grenzwerten hilfreich finden, die in der Vergangenheit dem EPALS-Course-Manual vorbehalten war ( $\triangleright$ Tab. 1). Außerdem wird neuerdings das „First-Hour-Management“ wichtiger pädiatrischer Notfälle besprochen.

Altersbereich: Unverändert gelten die Leitlinien zur Kinderreanimation bereits für Neugeborene, die die unmittelbare Geburtsphase hinter sich gelassen - also quasi den Kreißsaal verlassen haben. Interessant ist die diskrete Veränderung der oberen Altersgrenze: Alle Patienten bis zum 18. Lebensjahr werden wie Kinder reanimiert, es sei denn, man hält sie für Erwachsene.

Sauerstoff: Die Sauerstoffzufuhr wird so titriert, dass eine pulsoxymetrische Sätti- gung $\left(\mathrm{SpO}_{2}\right)$ von 94-98\% erreicht wird. Kann diese im Atem- und/oder Kreislaufversagen nicht gemessen werden, so wird Sauerstoff zunächst mit hohem Fluss appliziert.

Kreislauftherapie: Bei Kindern im Kreislaufversagen wird nunmehr ein Volumenbolus oder mehrere Boli von $10 \mathrm{ml} / \mathrm{kg}$ statt früher $20 \mathrm{ml} / \mathrm{kg}$ empfohlen. Nach jeder Volumengabe sollte der Volumenstatus reevaluiert werden - z. B. mittels Rekapillarisierungszeit. Der Einsatz vasoaktiver Substanzen soll frühzeitig erfolgen. Bei hämorrhagischem Schock sollten der Einsatz von Kristalloiden limitiert und möglichst früh Blutprodukte gegeben werden.

Beim Basic Life Support (BLS) sind einige Dinge klarer herausgestellt worden:

- BLS-Anwender sollten bei Abwesenheit eindeutiger Kreislaufzeichen umgehend nach den 5 Initialbeatmungen mit Thoraxkompressionen beginnen - früher wurden hier maximal 10 Sekunden Lebenszeichen geprüft.

- Im Zeitalter überall verfügbarer Smartphones geht man davon aus, dass auch ein einzelner Helfer parallel reanimieren und über Lautsprecher-Anruf den Notruf absetzen kann. Nur wenn kein Mobiltelefon verfügbar ist, wird empfohlen - wie früher - zunächst 1 Minute zu reanimieren und dann den Notruf zu veranlassen.

- Für alle entsprechend kompetenten Anwender ist die 2-Helfer-MaskenBeatmung der Standard, weil sie deutlich einfacher und sicherer ist.

Auch beim Advanced Life Support wurden nur Schwerpunkte verändert und die emp- fohlene Beatmungsfrequenz der Physiologie angepasst:

- Asynchrone Ventilationen mit einer altersadaptierten Beatmungsfrequenz (10-25/min) werden nur für intubierte Kinder empfohlen; bei Anwendung der Larynxmaske soll interponiert beatmet werden (15:2).

- Im Fokus der erweiterten Reanimation soll das Erkennen und Behandeln der reversiblen Ursachen („H’s \& HITS“) stehen.

- Im Zweifelsfall sollte der Rhythmus als schockbar klassifiziert werden.

- Für die Durchführung der trachealen Intubation werden nun nicht nur ein entsprechend erfahrener Anwender, sondern zusätzlich ein genau definiertes Verfahren und ein entsprechendes Equipment gefordert!

- Die Anwendung des DOPES-Akronyms wird auf alle Beatmungssituationen (Tubus, Larynxmaske, Maske) erweitert.

- Als Beispiele für supraglottische Atemwege werden nur Larynxmaske und I-Gel-Maske erwähnt.

Erfreulich ist, dass notfallmedizinische Innovationen - nicht zuletzt auch aus der deutschsprachigen Literatur - Eingang in die Leitlinien gefunden haben, wie z. B. Einsatz balancierter Kristalloide statt $\mathrm{NaCl}$ $0,9 \%$, frühzeitiger Einsatz von nichtinvasiver Beatmung, Gabe von Tranexamsäure bei schwerem Trauma, Larynxmaske als bevorzugter supraglottischer Atemweg, standardisierte Ausrüstung für Kindernotfälle, Verwendung der AVPU Scale und bevorzugte Thoraxpunktionsstelle im 4./5. ICR. VAL (z. B. [2, 3, 4, 5, 6]).

-Tab. 1 Normalwerte.

\begin{tabular}{|c|c|c|c|c|c|}
\hline Alter & 1 Monat & 1 Jahr & 2 Jahre & 5 Jahre & 10 Jahre \\
\hline Atemfrequenz $(1 / \mathrm{min})$ & $25-60$ & $20-50$ & $18-40$ & $17-30$ & $14-25$ \\
\hline Herzfrequenz (1/min) & $110-180$ & $100-170$ & $90-160$ & $70-140$ & $60-120$ \\
\hline syst. RR Untergrenze $(\mathrm{mmHg})$ & 50 & 70 & k.A. & 75 & 80 \\
\hline MAP Untergrenze $(\mathrm{mmHg})$ & 40 & 50 & k.A. & 55 & 55 \\
\hline
\end{tabular}




\section{Was ist (zu) alt?}

Bedauern werden einige Praktiker das Beharren auf der initialen $A B C-S e q u e n z$ für den Start der Basismaßnahmen. Hier hätte ein Weglassen der 5 Initialbeatmungen die Analogie zur Erwachsenen-CPR und damit die Handlungssicherheit verbessert - ohne den Zeitpunkt der ersten Beatmung wesentlich zu verzögern. Außerdem dauert vor allem im prähospitalen Setting die Vorbereitung der Beutel-Maske-Beatmung einige Sekunden, die für einen früheren Start der Thoraxkompressionen genutzt werden könnten.

Fast sämtliche Autor*innen arbeiten in spezialisierten pädiatrischen Einheiten vielleicht sollten hier zukünftig auch Vertreter*innen der nicht spezialisierten Notfallmedizin mitarbeiten?

Interessenkonflikt

Die Autorinnen/Autoren geben an, dass kein Interessenkonflikt besteht.
Autorinnen/Autoren

\section{Bernd Landsleitner ${ }^{1}$}

1 Dr. med., Abteilung für Anästhesie und Intensivmedizin, Cnopfsche Kinderklinik/ Klinik Hallerwiese Nürnberg

\section{Korrespondenzadresse}

Dr. med. Bernd Landsleitner

Abteilung für Anästhesie und

Intensivmedizin

Cnopfsche Kinderklinik/Klinik Hallerwiese

Nürnberg

St. Johannis-Mühlgasse 19

90419 Nürnberg

Deutschland

bernd.landsleitner@diakonieneuendettelsau. de

\section{Literatur}

[1] Van der Voorde P, Turner NM, Djakow J et al. European Resuscitation Council Guidelines 2021: Paediatric Life Support. Resucitation 2021. doi:10.1016/j.resuscitation.2021.02.0 15

[2] Hoffmann F, Schmalhofer M, Lehner $\mathrm{M}$ et al. Comparison of the AVPU Scale and the Pediatric GCS in Prehospital Setting. Prehosp Emerg Care 2016; 20: 493-498
[3] Arbeitsgemeinschaft der Wissenschaftlichen Medizinischen Fachgesellschaften e.V. (AWMF). AWMF-Leitlinie 001/032. Perioperative Infusionstherapie bei Kindern. 2016. Im Internet (Stand: 27.04.2021): https://www. awmf.org/uploads/tx_szleitlinien/001032I_S1_S1_Infusionstherapie_perioperativ_Kinder_2016-02-abgelaufen.pdf

[4] Terboven T, Leonhard G, Wessel L et al. Chest wall thickness and depth to vital structures in paediatric patients - implications for prehospital needle decompression of tension pneumothorax. Scand J Trauma Resusc Emerg Med 2019; 27: 45

[5] Keil J, Jung P, Schiele A et al. Interdisziplinär konsentierte Stellungnahme zum Atemwegsmanagement mit supraglottischen Atemwegshilfen in der Kindernotfallmedizin. Anästhesist 2016; 65: 57-66

[6] Timmermann A, Böttiger BW, Byhahn C et al. S1-Leitlinie Prähospitales Atemwegsmanagement. Anästh Intensivmed 2019; 60: 316336

\section{Bibliografie}

Notarzt 2021; 37: 146-147

online publiziert 11.05.2021

DOI 10.1055/a-1448-8043

ISSN 0177-2309

(c) 2021. Thieme. All rights reserved.

Georg Thieme Verlag KG, Rüdigerstraße 14, 70469 Stuttgart, Germany 